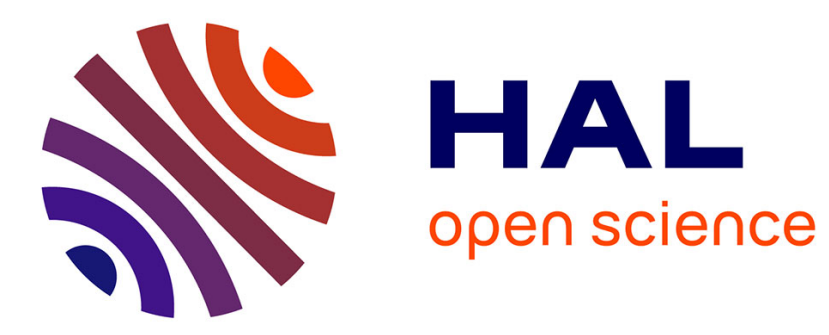

\title{
Addressing factors fixing setting from given data: A comparison of different methods
}

Thierry A. Mara, Benjamin Belfort, Vincent Fontaine, Anis Younes

\section{To cite this version:}

Thierry A. Mara, Benjamin Belfort, Vincent Fontaine, Anis Younes. Addressing factors fixing setting from given data: A comparison of different methods. Environmental Modelling and Software, 2017, 87, pp.29 - 38. 10.1016/j.envsoft.2016.10.004 . hal-01398207

\section{HAL Id: hal-01398207 https://hal.science/hal-01398207}

Submitted on 16 Nov 2016

HAL is a multi-disciplinary open access archive for the deposit and dissemination of scientific research documents, whether they are published or not. The documents may come from teaching and research institutions in France or abroad, or from public or private research centers.
L'archive ouverte pluridisciplinaire HAL, est destinée au dépôt et à la diffusion de documents scientifiques de niveau recherche, publiés ou non, émanant des établissements d'enseignement et de recherche français ou étrangers, des laboratoires publics ou privés. 


\title{
Addressing factors fixing setting from given data: A comparison of different methods
}

\author{
Thierry A. Mara ${ }^{\mathrm{a}, *}$, Benjamin Belfort ${ }^{\mathrm{b}}$, Vincent Fontaine ${ }^{\mathrm{a}}$, Anis Younes ${ }^{\mathrm{b}, \mathrm{c}}$ \\ ${ }^{a}$ PIMENT, EA 4518, Université de La Réunion, FST, 15 Avenue René Cassin, 97715 \\ Saint-Denis, Réunion \\ ${ }^{b}$ LHyGeS, UMR-CNRS 7517, Université de Strasbourg/EOST, 1 rue Blessig, 67084 \\ Strasbourg, France \\ ${ }^{c}$ IRD UMR LISAH, F-92761 Montpellier, France
}

\begin{abstract}
This paper deals with global sensitivity analysis of computer model output. Given a set of independent input sample and associated model output vector with possibly the vector of output derivatives with respect to the input variables, we show that it is possible to evaluate the following global sensitivity measures: (i) the Sobol' indices, (ii) the Borgonovo's density-based sensitivity measure, and (iii) the derivative-based global sensitivity measure of Sobol' and Kucherenko. We compare the efficiency of the different methods to address factors fixing setting, an important issue in global sensitivity analysis. First, global sensitivity analysis of the Ishigami function is performed with the different methods. Then, they are applied to two different responses of a soil drainage model. The results show that the polynomial chaos expansion for estimating Sobol' indices is the most efficient approach.

Keywords: factors fixing setting, variance-based sensitivity measure, density-based sensitivity measure, derivative-based global sensitivity measure, simulated drainage experiment
\end{abstract}

\footnotetext{
${ }^{*}$ Corresponding author: mara@univ-reunion.fr
} 


\section{Contents}

1 Introduction 3

2 Global sensitivity analysis $\quad 5$

2.1 Variance-based sensitivity measures . . . . . . . . . . 5

2.2 Density-based sensitivity measure . . . . . . . . . . 8

2.3 Derivative-based global sensitivity measure . . . . . . . . . . . 10

2.4 GSA setting . . . . . . . . . . . . . . . . . . 12

3 Numerical test: the Ishigami function 12

4 Application to an unsaturated flow model 14

4.1 Drainage experiment . . . . . . . . . . . . . . . . 14

4.2 GSA of the pressure head . . . . . . . . . . . . 16

4.3 GSA of the average water content . . . . . . . . . . 18

$\begin{array}{lll}5 & \text { Summary and conclusion } & 20\end{array}$ 


\section{Introduction}

Computer models are widely used to mimic systems and processes of different natures (e.g. physical, environmental or economical) because running a computer model is less expensive than performing an experimental set-up. Computer models can help understanding the process under study and/or improving its management. However, the different sources of uncertainty in the computer models should be taken into account in order to avoid erroneous inferences. For this purpose, the functional inputs should be treated as stochastic processes and the uncertain scalar inputs as random variables defined by a joint probability density function (see Blatman and Sudret, 2011; Anstett-Collin et al., 2015). As a consequence, computer model predictions are random quantities too.

Both uncertainty and sensitivity analyses are helpful for measuring the impact of the different sources of uncertainty in the model response of interest. Saltelli et al. (2006) note that the response of interest "should not be concerned with the model output per se, but with the question that the model has been called to answer." While uncertainty analysis aims at assessing the uncertainty of model output, global sensitivity analysis (GSA) aims at pinpointing the different sources of uncertainty of the model response uncertainty. There are two situations to consider when GSA is to be undertaken: the case of independent inputs and the case of dependent inputs. The latter can be challenging (see for instance, Kucherenko et al., 2012; Mara and Tarantola, 2012; Mara et al., 2015), depending on the question addressed (see some possible issues in Saltelli and Tarantola, 2002). Generally, prior to a thorough data collection and statistical calibration of the computer model, independence of the inputs can be reasonably assumed. In the present work, independent input scalar variables are considered. 
Let $y=f(\boldsymbol{x})$ be the scalar response of a computer model function of $d$ independent random variables $\boldsymbol{x}=\left(x_{1}, \ldots, x_{d}\right) \sim p_{1}\left(x_{1}\right) \times \cdots \times p_{d}\left(x_{d}\right)$. We also consider the vector of local sensitivities $\boldsymbol{j}=\left(j_{1}, \ldots, j_{d}\right)$ (with $\left.j_{i}=\partial y / \partial x_{i}\right)$ possibly provided by the computer model itself. We denote by $\mathbf{X}$, a $N \times d$ matrix, a given sample of $\boldsymbol{x}$. The vector of responses associated to $\mathbf{X}$ is denoted $\mathbf{y}$ and $\mathbf{J}$ is the matrix of partial derivatives (also called the Jacobian matrix) obtained after evaluating the computer model with each of the $N$ independent draws. The availability of the Jacobian matrix is only compulsory for the assessment of the so-called derivative-based global sensitivity measure (see below). It is not mandatory for the two other sensitivity measures studied in this work.

Recent reviews on sensitivity analysis have focused primarily on methods employing specific sampling designs (e.g., Iooss, 2011; Saltelli et al., 2012; Neumann, 2012; Norton, 2015; Borgonovo and Plischke, 2015, among others). In the present article, we address how to pinpoint the relevant input variables from a given set of data $(\mathbf{X}, \mathbf{y}, \mathbf{J})$. Our aim is to demonstrate that, with a single sample of size $N$ (say, thousands), several global sensitivity indices can be evaluated. Three global sensitivity measures are studied, namely: (i) the Sobol' indices (Sobol', 1993; Homma and Saltelli, 1996), (ii) the sensitivity measure of Borgonovo (2006), and (iii) the derivative-based global sensitivity measure (Lamboni et al., 2013). These sensitivity indices are of different natures and may lead to different inferences about the model input importance.

The choice of the sensitivity index to compute and the model output to consider depends on the question that the model has been called to answer (Saltelli et al., 2004). In this work, we address the factors fixing setting (henceforth, FF setting) with the different sensitivity measures. Saltelli et al. 
(2006) define this setting as follows: "The objective of this setting, which could also be labelled as screening, is to identify the input factor or the subset of input factors that we can fix at any given value over their range of uncertainty without reducing significantly the output variance." One could even require " ... without changing significantly the predicted output probability density function."

There are many sensitivity measures proposed in the literature to address the FF setting. Compared to those studied in the present work, some of them are of higher-order (e.g., Owen et al., 2014; Gamboa et al., 2015). In our study, we focus on the three aforementioned sensitivity indices because they are of different natures (i.e. variance-based, density-based and derivativebased sensitivity measures respectively) and can be assessed from a single sample. Plischke et al. (2013) define other possible moment-independent importance measures which can be estimated from given data. Their estimation is based on a method similar to the one described in this paper for assessing Borgonovo's importance measure (see Section 2.2). Therefore, we only consider the Borgonovo's importance measure in our study.

In the present work, we do not consider GSA methods based on surrogate models that are run to assess the sensitivity indices. The reasons are i) there is a plethora of different emulators, and it is not the point here to discuss their merits and flaws and ii) some emulators can require specific experimental designs for the sake of efficiency (e.g. the Gaussian emulator Oakley and O'Hagan, 2004), while it is assumed here that the sample is arbitrary (though independent). It can be notified that the polynomial chaos expansion method described in Section 2.1 is a surrogate-based approach. However, we argue that this approach employs ANOVA-like decomposition and as such is classified amongst the spectral methods (such as the 
Fourier amplitude sensitivity test, Cukier et al., 1973; Schaibly and Shuler, 1973; Saltelli et al., 1999). Indeed, as detailed later (see Section 2.1), the variance-based sensitivity indices are directly obtained from the regression coefficients without needing to run the surrogate model.

The paper is organized as follows: In Section 2 we discuss the global SA methods based on the Sobol' indices (Section 2.1), the density-based importance measure of Borgonovo (Section 2.2), and the derivative-based global sensitivity measure (Section 2.3). First, their performance for addressing factors fixing setting of a well-known non-linear function is studied in Section 3. Then, the different methods are applied to perform GSA of a soil drainage experiment in Section 4. Finally, a summary and conclusions are given in Section 5 .

\section{Global sensitivity analysis}

\subsection{Variance-based sensitivity measures}

Variance-based sensitivity indices (also called Sobol' indices) are the most popular importance measures for GSA. By computing all Sobol' indices (there are $2^{d}-1$ indices at most), one can guess the structure of the input/output relationship. In practice, two variance-based sensitivity indices are particularly interesting: the first-order sensitivity index $S_{i}$ (also called correlation ratio by Pearson, 1905; McKay, 1996) and the total sensitivity index $S T_{i}$ (first defined in Homma and Saltelli, 1996). They are defined as follows:

$$
\begin{aligned}
& S_{i}=\frac{\mathbb{V}\left[\mathbb{E}\left[y \mid x_{i}\right]\right]}{\mathbb{V}[y]} \in[0,1] \\
& S T_{i}=\frac{\mathbb{E}\left[\mathbb{V}\left[y \mid \boldsymbol{x}_{\sim i}\right]\right]}{\mathbb{V}[y]} \in[0,1]
\end{aligned}
$$


where $\boldsymbol{x}_{\sim i}=\boldsymbol{x} \backslash x_{i} . \mathbb{E}[\cdot]$ and $\mathbb{V}[\cdot]$ represent the expectation and variance operators, respectively. $\mathbb{E}[\cdot \mid \cdot]$ and $\mathbb{V}[\cdot \mid \cdot]$ represent the conditional expectation and conditional variance operators, respectively.

$S_{i}$ measures the amount of variance of $y$ due to the uncertainty of $x_{i}$ alone, while $S T_{i} \geq S_{i}$ measures the amount of all contributions of $x_{i}$ to the variance of $y$, including its cooperative non-linear contributions with the other variables gathered in $\boldsymbol{x}_{\sim i}$. The higher $S T_{i}$ is, the more $x_{i}$ is an important input for the variance of $y$. The input/output relationship is additive when $S_{i}=S T_{i}, \forall i=1, \ldots, d$ and, in this case, $\sum_{i=1}^{d} S_{i}=1$.

Sobol' indices were introduced in the framework of ANOVA-HDMR (acronym for analysis of variance - high dimensional model representation, see Rabitz et al., 1999). The latter relies on the existence of a unique ANOVA representation of the input/output relationship when model inputs are independent (otherwise, such a representation is not unique, see for instance Mara et al., 2015). Thus, the $2^{d}-1$ Sobol' indices reflect the structure of the model response. This is one of the appealing features of Sobol' indices. Indeed, $\left(1-\sum_{i=1}^{d} S_{i}\right)$ represents the amount of variance due to interactions between the input variables.

There are several computationally affordable methods proposed in the literature to compute the set of first-order sensitivity indices (Oakley and O'Hagan, 2004; Tarantola et al., 2006; Ratto et al., 2007; Lewandowski et al., 2007; Mara and Joseph, 2008; Plischke, 2010, among others). However, it is much more expensive to compute the entire set of total sensitivity indices. From given data, this task can be achieved with sparse polynomial chaos expansions (Blatman and Sudret, 2010, 2011; Fajraoui et al., 2012; Shao et al., 2016). ANOVA-HDMR presumes that $f(\boldsymbol{x})$ is square-integrable. This means that 
$f \in \mathcal{H}$, where $\mathcal{H}$ is a Hilbert space equipped with the following inner-product:

$$
<f, g>_{\mathcal{H}}=\mathbb{E}[f(\boldsymbol{x}) g(\boldsymbol{x})]
$$

with $g \in \mathcal{H}$. Polynomial chaos expansion (PCE) is a spectral method that casts the continuous scalar model response onto orthonormal polynomials as follows:

$$
f(\boldsymbol{x})=\sum_{\boldsymbol{\alpha} \subset \mathbb{N}^{d}} a_{\boldsymbol{\alpha}} \Psi_{\boldsymbol{\alpha}}(\boldsymbol{x})
$$

where $\boldsymbol{\alpha}=\alpha_{1} \alpha_{2} \ldots \alpha_{d} \in \mathbb{N}^{d}$ is a $d$-dimensional index and the $a_{\boldsymbol{\alpha}}$ s are the PCE coefficients to be determined. The $\Psi_{\alpha}$ s are the multidimensional polynomial basis elements of degree $|\boldsymbol{\alpha}|=\sum_{i=1}^{d} \alpha_{i}$. They form a complete orthonormal basis in $\mathcal{H}$. For some specific random variables, they are derived from the Askey scheme (see Askey and Wilson, 1985; Xiu and Karniadakis, 2002). In the case of a vector of arbitrarily distributed variables $\boldsymbol{x}$, one can either resort to isoprobabilistic transformations (see Sudret, 2008) or build-up the orthonormal polynomials with the data-driven method of Oladyshkin and Novak (2012) or alternatively the stable procedure of Stieltjes (see Gautschi, 1982, 1993). Because the numerical exercise in Section 3 and the application in Section 4 deal with parameters uniformly distributed, throughout this paper Legendre polynomials are employed in expansion (3).

The multidimensional polynomial $\Psi_{\boldsymbol{\alpha}}$ is simply the tensor product of univariate polynomials,

$$
\Psi_{\boldsymbol{\alpha}}(\boldsymbol{x})=\prod_{i=1}^{d} \psi_{\alpha_{i}}\left(x_{i}\right)
$$

with $\psi_{0}=1$.

Due to the orthonormality of the polynomial basis elements, the total variance of $f(\boldsymbol{x})$ reduces to the sum of squares of the PCE coefficients,

$$
\mathbb{V}[y]=\sum_{|\boldsymbol{\alpha}|>0}^{+\infty} a_{\boldsymbol{\alpha}}^{2}
$$


and the conditional expectation of $y$ onto $x_{i}$, also called $x_{i}$ 's marginal effect, writes

$$
\mathbb{E}\left[y \mid x_{i}\right]=a_{0 \ldots 0}+\sum_{\alpha_{i}>0}^{+\infty} a_{0 \ldots \alpha_{i} \ldots 0} \psi_{\alpha_{i}}\left(x_{i}\right) .
$$

For practical reasons, the previous expansions are truncated up to a prescribed degree $p$. The resulting PCE coefficients are then evaluated by using a regression method yielding

$$
\hat{\mathbf{a}}=\underset{\boldsymbol{a}}{\operatorname{argmin}}\left\{\sum_{k=1}^{N}\left(y_{k}-\sum_{|\boldsymbol{\alpha}| \leq p} a_{\boldsymbol{\alpha}} \Psi_{\boldsymbol{\alpha}}\left(X_{k 1}, \ldots, X_{k d}\right)\right)^{2}\right\}
$$

where $\hat{\boldsymbol{a}}$ is the vector of estimated coefficients and $\left(X_{k 1}, \ldots, X_{k d}\right)$ is the $k$-th draw of the input vector.

The first-order and total sensitivity indices of $x_{i}$ are then inferred as follows:

$$
\begin{aligned}
\hat{S}_{i}^{P C E} & =\frac{\sum_{\alpha_{i}>0}^{p} \hat{a}_{0 \ldots \alpha_{i} \ldots 0}^{2}}{\sum_{|\boldsymbol{\alpha}|>0}^{p} \hat{a}_{\boldsymbol{\alpha}}^{2}} \\
\hat{S T}_{i}^{P C E} & =\frac{\sum_{\boldsymbol{\alpha}: \alpha_{i}>0}^{p} \hat{a}_{\boldsymbol{\alpha}}^{2}}{\sum_{|\boldsymbol{\alpha}|>0}^{p} \hat{a}_{\boldsymbol{\alpha}}^{2}}
\end{aligned}
$$

Thus, evaluating the variance-based sensitivity indices with PCE amounts to estimating the polynomial coefficients. For a PCE of degree $p$, there are $(p+d) ! / p ! d$ ! coefficients to compute. However, most of these coefficients are not significant regarding the total variance. Hence, it is advisable to discard the irrelevant terms in the estimated truncated PCE. This leads to the so-called sparse PCE (Blatman and Sudret, 2010). There are several algorithms proposed in the literature to build-up a sparse PCE 
(e.g. Blatman and Sudret, 2011; Fajraoui et al., 2012). The one proposed in Shao et al. (2016) is used in the present work. The approach developed in Shao et al. (2016) generally provides very sparse truncated PCEs because of the specific algorithm employed. The latter combines a model selection criterion with informative prior to infer the optimal sparse PCE in a Bayesian framework. The prior is chosen in order to favour low degrees and low interaction terms. The MATLAB programs are available upon request from the first author of the present paper. Another alternative is to use UQLab, the MATLAB toolbox developed by Marelli and Sudret (2014).

\subsection{Density-based sensitivity measure}

The development of density-based sensitivity measures was motivated by the fact that variance-based sensitivity measures only reveal the relevant inputs for the output variance, which is only the second-order moment of the probability density function of the output. Hence, some authors have advocated the use of moment-independent importance measure (Chun et al., 2000; Borgonovo, 2006, 2007). Several moment-independent sensitivity measures have been proposed in recent literature (some of them are described in Plischke et al., 2013; Borgonovo et al., 2016). In the present work, we consider the density-based sensitivity measure introduced by Borgonovo (2006). The sensitivity index of Borgonovo measures the importance of the input variable over the entire pdf and is defined as follows:

$$
\delta_{i}=\frac{1}{2} \mathbb{E}\left[s\left(x_{i}\right)\right] \in[0,1]
$$

with,

$$
s\left(x_{i}\right)=\int_{\mathbb{R}}\left|p_{y}(y)-p_{y \mid x_{i}}\left(y \mid x_{i}\right)\right| \mathrm{d} y
$$

where $p_{y}(y)$ is the probability density function (pdf) of $y$ and $p_{y \mid x_{i}}\left(y \mid x_{i}\right)$ is the conditional pdf of $y$ onto $x_{i}$. It is worth noting that $s\left(x_{i}\right)$ measures the 
area between $p_{y}$ and $p_{y \mid x_{i}}$ (see Figure 1) so that the latter is null if $p_{y \mid x_{i}}=p_{y}$. Eq. (8) simply computes the half expectation of $s\left(x_{i}\right)$ with respect to $x_{i}$. If $\delta_{i}=0$ then fixing $x_{i}$ to any value in its uncertainty range does not change the predicted pdf.

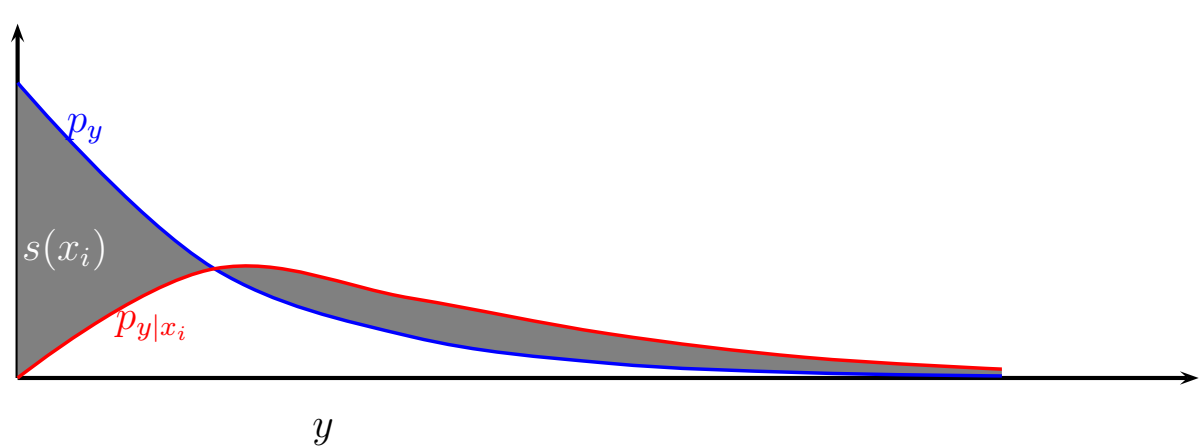

Figure 1: In blue, the unconditional predicted pdf $\left(p_{y}\right)$ is represented. The red curve is the conditional predicted pdf $\left(p_{y \mid x_{i}}\right)$. The shaded area $s\left(x_{i}\right)$ measures the shift between the two densities.

Borgonovo importance measure (BIM) is a strong measure of statistical dependence between two random variables (Strong and Oakley, 2013). As opposed to Sobol' indices, BIM does not require the existence of an HDMR. Thus, because $\delta_{i}$ only measures the pairwise dependency between $\left(x_{i}, y\right)$, its definition is somehow simpler than $S T_{i}$. Meanwhile, as shown in the following numerical exercises, accurate estimate of BIM may require a large simple size. But, as also shown, rough estimate of the BIMs at modest sample size (say one thousand) may be sufficient for detecting irrelevant inputs. This is a desired feature for screening purposes of high-dimensional computer models.

Up to 2013, the use of BIM for GSA of computer models was hampered by its computational cost. Indeed, it can be noticed that, for each input, a double loop is necessary to assess its density-based sensitivity measue (Eqs. $(8-9))$. In the first loop, the current variable $x_{i}$ is set to prescribed values, 
while in the second loop several draws of the remaining inputs $\boldsymbol{x}_{\sim i}$ are generated. For each prescribed value, an output vector is obtained from which the conditional pdf (onto $x_{i}$ ) can be inferred. However, by noticing that setting $x_{i}$ to different values is almost the same as varying $x_{i}$ within a narrow range, Plischke et al. (2013) proposed a cheaper computational method to estimate the BIM of each input variable with only one single sample (i.e. one single loop). Therefore, the proposed approach alleviates the so-called curse of dimensionality. The principle of the approach is explained hereafter.

In the approach proposed by Plischke et al. (2013), the draws of $x_{i}$ denoted $\left\{X_{k i}, k=1, \ldots, N\right\}$ are partitioned into $M$ subsamples of equal size $\left(\mathbf{X}_{i}^{1}, \ldots, \mathbf{X}_{i}^{M}\right)$. It must be ensured that there are enough draws in each partition for an accurate estimation of the conditional pdf (say a hundred). In the case of equiprobable partitions, the number of draws per partition is $P=N / M$. Such a partitioning can be easily understood with a pairwise scatterplot (see the top plot of Figure 2). Then, a rough estimation of $s\left(x_{i}\right)$ (see Eq. (9)) for $x_{i}=\bar{x}_{i}^{1}=\sum_{k=1}^{P} X_{k i}^{1} / P$ is,

$$
\hat{s}\left(\bar{x}_{i}^{1}\right)=\int_{\Omega_{y}}\left|\hat{p}_{y}(y)-\hat{p}_{y \mid x_{i}}\left(y \mid x_{i} \in \mathbf{X}_{i}^{1}\right)\right| \mathrm{d} y
$$

where $\Omega_{y}$ denotes the range of variation of $\mathbf{y}$.

Numerically, Eq. (10) is computed by comparing the estimated pdf of $\mathbf{y}$ (i.e. $\hat{p}_{y}$ ) with the one of $\mathbf{y}_{i}^{1}$ (i.e. $\hat{p}_{y \mid x_{i}}$ for $x_{i}=\bar{x}_{i}^{1}$ ). This is illustrated in the bottom plot of Figure 2 for each partition. The smoothing kernel-density approach (Parzen, 1962) is used by the authors to assess the pdfs from the sample and subsamples. In order to reduce bias in the estimate of the BIM, it must be ensured that $\hat{p}_{y}(y)$ and $\hat{p}_{y \mid x_{i}}\left(y \mid x_{i}=\bar{x}_{i}^{1}\right)$ are significantly different. For this purpose, the Kolmogrov-Smirnov's test is employed (Press et al., 2007).

Finally, the calculation of $\delta_{i}$ is straightforward because the partitioning 
is equiprobable, and the result is

$$
\hat{\delta}_{i}=\frac{1}{2 M} \sum_{j=1}^{M} \hat{s}\left(\bar{x}_{i}^{j}\right) .
$$

The choice of $M$ is one of the degrees of freedom of the proposed approach. Given that each subsample must have a sufficient number of draws (say hundreds) to accurately infer the conditional pdf, the choice of $M$ depends on the sample size $N$. In Section 3, we numerically show that, for small sample sizes, BIM estimates are not accurate but can allow for detecting irrelevant input variables. In the present work, the BIMs are estimated with the program developed by E. Plischke and available on the website of the Joint Research Center (https://ec.europa.eu/jrc/en/samo/simlab).

\subsection{Derivative-based global sensitivity measure}

Until the end of the last century, local sensitivity indices were intensively used to perform sensitivity analysis of a given model response $y=f(\boldsymbol{x})$. These indices rely on partial derivatives of the model response evaluated at some point $\boldsymbol{x}^{*}$. This point generally reflects the analyst's best knowledge about the input values. The success of local SA is partially explained by the usefulness of partial derivatives in model calibration and the existence of efficient computational methods to assess the sensitivity matrix (e.g. automatic differentiation, adjoint method Cacuci, 1981; Griewank, 1989; Navon, 1997). The local sensitivity index of $x_{i}$ at $\boldsymbol{x}^{*}$ is defined as follows:

$$
L S_{i}=\left.x_{i}^{*} \frac{\partial y}{\partial x_{i}}\right|_{\boldsymbol{x}^{*}}
$$

The higher is $L S_{i}$, the more $x_{i}$ is locally relevant.

Local sensitivity analysis suffers at least from two drawbacks: (i) the result is local and will differ from one point to another for non-linear functions 

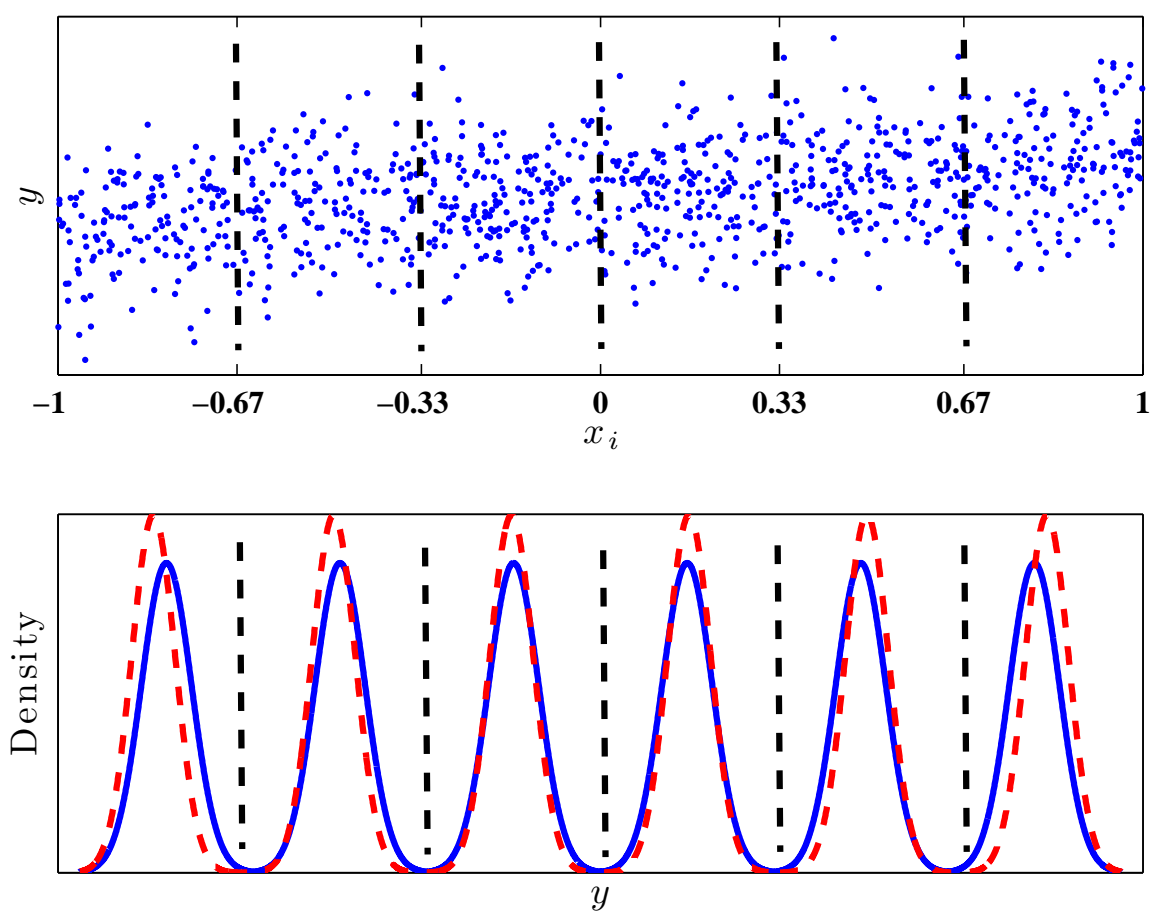

Figure 2: On the top, the scatterplot of the model response $y$ versus the input variable $x_{i}$. The draws are partitioned into $M=6$ subsamples of equal size (here $x_{i}$ is uniformly distributed). On the bottom, the pdf of the total draws of $y$ (blue continuous curve) is compared to the conditional pdf of the draws localized in the partitions (red broken line).

and (ii) it does not capture the possible interactions amongst the inputs. To circumvent these drawbacks, a derivative-based global sensitivity measure (DGSM) has been defined, thanks to the recent work of Lamboni et al. (2013) and the earlier works of Sobol' and Kucherenko (2009, 2010). DGSM is inferred by evaluating the partial derivatives at several points in the input space. Indeed, in Lamboni et al. (2013) the authors define the following statistic:

$$
\nu_{i}=\int_{\mathbb{R}^{d}}\left(\frac{\partial y}{\partial x_{i}}\right)^{2} \prod_{j=1}^{d} p_{j}\left(x_{j}\right) d x_{j} .
$$


The authors then show that,

$$
S T_{i} \leq 4 C_{i}^{2} \frac{\nu_{i}}{\mathbb{V}[y]}
$$

where $C_{i}$ is called the Cheeger constant defined as follows:

$$
C_{i}=\sup _{x_{i} \in \mathbb{R}} \frac{\min \left(F_{i}\left(x_{i}\right), 1-F_{i}\left(x_{i}\right)\right)}{p_{i}\left(x_{i}\right)}
$$

with $F_{i}\left(x_{i}\right)$ the cumulative distribution function of $x_{i}$ (i.e. $\left.p_{i}\left(x_{i}\right)=\frac{\mathrm{d} F_{i}}{\mathrm{~d} x_{i}}\right)$.

The Cheeger constant has been defined by Sobol' and Kucherenko (2009, 2010) for the uniform distribution $p_{i}\left(x_{i}\right)=\mathcal{U}\left(a_{i}, b_{i}\right)$ as $C_{i}=\frac{\left(b_{i}-a_{i}\right)}{2 \pi}$ and for the normal distribution $p_{i}\left(x_{i}\right)=\mathcal{N}\left(\mu_{i}, \sigma_{i}^{2}\right)$ as $C_{i}=\frac{\sigma_{i}}{2}$. This constant can be computed for any distribution that belongs to the class of Boltzmann probability measure (see Lamboni et al., 2013, for more details).

From Eq. (14), it can be inferred that if the DGSM $\Upsilon_{i}=4 C_{i}^{2} \frac{\nu_{i}}{\mathbb{V}[y]}$ is small, $x_{i}$ is an irrelevant input. Finally, we note that from a given (quasi) Monte Carlo sample of partial derivatives matrix $\mathbf{J}, \nu_{i}$ is estimated as follows:

$$
\hat{\nu}_{i}=\frac{1}{N} \sum_{k=1}^{N} J_{k i}^{2} .
$$

It is then straightforward to get a (quasi) Monte Carlo estimate of $\Upsilon_{i}$.

\subsection{GSA setting}

Comparing different importance measures can be misleading because "importance" is not a mathematical concept (Saltelli et al., 2004). Saltelli et al. (2006) advice to define "importance" at the stage of framing the analysis. We note that DGSM is particularly suited for screening purposes, which consists of investigating unessential inputs. Hence, in the present work, we study the ability of the different methods to pinpoint the irrelevant inputs of a given model response. This is known as the factors fixing setting (Saltelli and Tarantola, 2002). 
We particularly investigate whether the different methods are subject to Type I error (i.e. failing at identifying irrelevant inputs) or Type II error (i.e. identifying influential inputs as irrelevant ones). It is expected that the risk of error is high for small sample sizes. We can also expect that performing FF setting with DGSM might be subject to Type I error because it is an upper bound for the total sensitivity index (see Eq. (14)). Thereby, it is only informative when its value is very small (for irrelevant inputs).

\section{Numerical test: the Ishigami function}

GSA is performed on the Ishigami function defined as,

$$
f\left(x_{1}, x_{2}, x_{3}, x_{4}\right)=\sin \left(x_{1}\right)+7 \sin ^{2}\left(x_{2}\right)+0.1 x_{3}^{4} \sin \left(x_{1}\right)+\epsilon x_{4}
$$

with the input variables uniformly distributed as follows $x_{i} \sim \mathcal{U}(-\pi, \pi)$ and $\epsilon=10^{-6}$. This function is non-monotonic and includes a strong interaction between $x_{1}$ and $x_{3}$. Regarding FF setting, the first three input variables are important while $x_{4}$ is a non important parameter introduced in the analysis to make sure that the different methods are able to identify it as an irrelevant input.

The analysis is performed with quasi-Monte Carlo (QMC) sequences of different sizes, $N=\{64,128,256,512,1024,2048,4096,8192,16384\}$. For this purpose, we used the MATLAB program LPtau51.m developed by Sobol' et al. (1992) and downloadable on the website of the Joint Research Center (see the link before $\S 2.3$ ). BIM cannot be computed for small sample sizes because one must ensure that enough draws are contained in each subsample. We assign the following number of partitions to each sample $M=$ $\{0,0,2,4,8,16,16,16,16,32\}$, with $M=0$ meaning that BIM cannot be assessed. To estimate the DGSMs, the partial derivatives evaluated at the 
different QMC draws are also computed, namely,

$$
\begin{aligned}
& j_{1}(\boldsymbol{x})=\left(1+0.1 x_{3}^{4}\right) \cos \left(x_{1}\right) \\
& j_{2}(\boldsymbol{x})=14 \cos \left(x_{2}\right) \sin \left(x_{2}\right) \\
& j_{3}(\boldsymbol{x})=0.4 x_{3}^{3} \sin \left(x_{1}\right) \\
& j_{4}(\boldsymbol{x})=\epsilon
\end{aligned}
$$

The estimated sensitivity measures are plotted in Figure 3 versus $\log _{2}(N)=$ $6, \ldots, 14$. The results are compared with the analytical values. The latter are exactly calculated for the Sobol' indices. As far as the two other sensitivity measures are concerned, they are estimated from a QMC sample of size $N=2^{19}$. For the DGSM, a total of $M=64$ partitions are selected which is much higher than what is recommended in practice (see Plischke et al., 2013). This choice is done to get accurate estimates.

Figure 3a-b show that the variance-based sensitivity estimates are virtually constant. Actually, the PCE-based estimator have converged toward the analytical values proving that even for $N=64$ the sparse PCEs are good approximations of the original function. Such a fast convergence is due to the fact that the Ishigami function is very smooth (infinitely derivable w.r.t. all the input variables). Note that, because the input variables are uniformly distributed, the sparse PCE was built with Legendre polynomials. The first-order sensitivity indices indicate that $x_{2}$ has the higher first-order effect while the one of $x_{3}$ is zero. This means that it is expected that by fixing $x_{2}$ one has more chance to reduce the output variance than fixing $x_{3}$ (Saltelli and Tarantola, 2002). The total sensitivity indices indicate that $\left(x_{1}, x_{2}, x_{3}\right)$ are important and that $x_{4}$ is irrelevant.

It is worth mentioning that for $N=16384$, a 16-th degree polynomial has been identified with 26 terms in the expansion. A very sparse PCE is 
obtained due to i) the specific algorithm used (see Shao et al., 2016) and ii) the features of the Ishigami function. Indeed, the Ishigami function is odd with respect to $x_{1}$, even with respect to $x_{2}$ and the interaction term between $x_{1}$ and $x_{3}$ is the product of an even polynomial function of $x_{3}$ and an odd function of $x_{1}$. The identified sparse PCE inherits from these features and contains only odd degree monomials of $x_{1}$, even degree monomials of $x_{2}$ and products of odd degree monomials of $x_{1}$ with even degree monomials of $x_{3}$.

Figure 3c depicts the BIM estimates. We can note that the estimations have not completely converged even after $N \geq 16384$, especially for $\delta_{3}$ and $\delta_{2}$. This difference of convergence from one input to another seems to indicate that the partitioning should be adapted to the input variable for an overall better convergence of the estimator. At low sample sizes (i.e. $N \leq 512$ ) the approach is not able to successfully identify the three first inputs as important (Type II error). Although the convergence is slow, when the sample size is sufficiently large (here from $N=1024$ ) the estimator of Plischke et al. (2013) is able to detect the relevant inputs.

Regarding the DGSMs, Figure 3d shows that the estimates are quite stable from $N \geq 256$. We note that $\left(\hat{\Upsilon}_{1}, \hat{\Upsilon}_{2}, \hat{\Upsilon}_{3}\right)$ are greater than one which indicates that $\left(x_{1}, x_{2}, x_{3}\right)$ cannot be deemed as irrelevant. Note that the fact $\hat{\Upsilon}_{i}$ can be greater than one contrasts with the two other sensitivity measures scaled within $[0,1]$. Anyway, with the DGSM only $x_{4}$ is identified as non $\operatorname{important}\left(\hat{\Upsilon}_{4}=0\right)$.

For this exercise all the estimated sensitivity measures are able to detect the irrelevant input $x_{4}$. PCE-based total sensitivity indices and DGSMs require less computational effort (i.e. draws) to effectively identify the irrelevant inputs. Note that because the sensitivity measures are based on different definitions, they can yield different rankings of importance. Indeed, accord- 
ing to Figure 3, the input vector ranked by order of importance is $\left(x_{1}, x_{2}, x_{3}\right)$ with the total sensitivity index, $\left(x_{2}, x_{1}, x_{3}\right)$ with BIM and $\left(x_{2}, x_{3}, x_{1}\right)$ with DGSM.
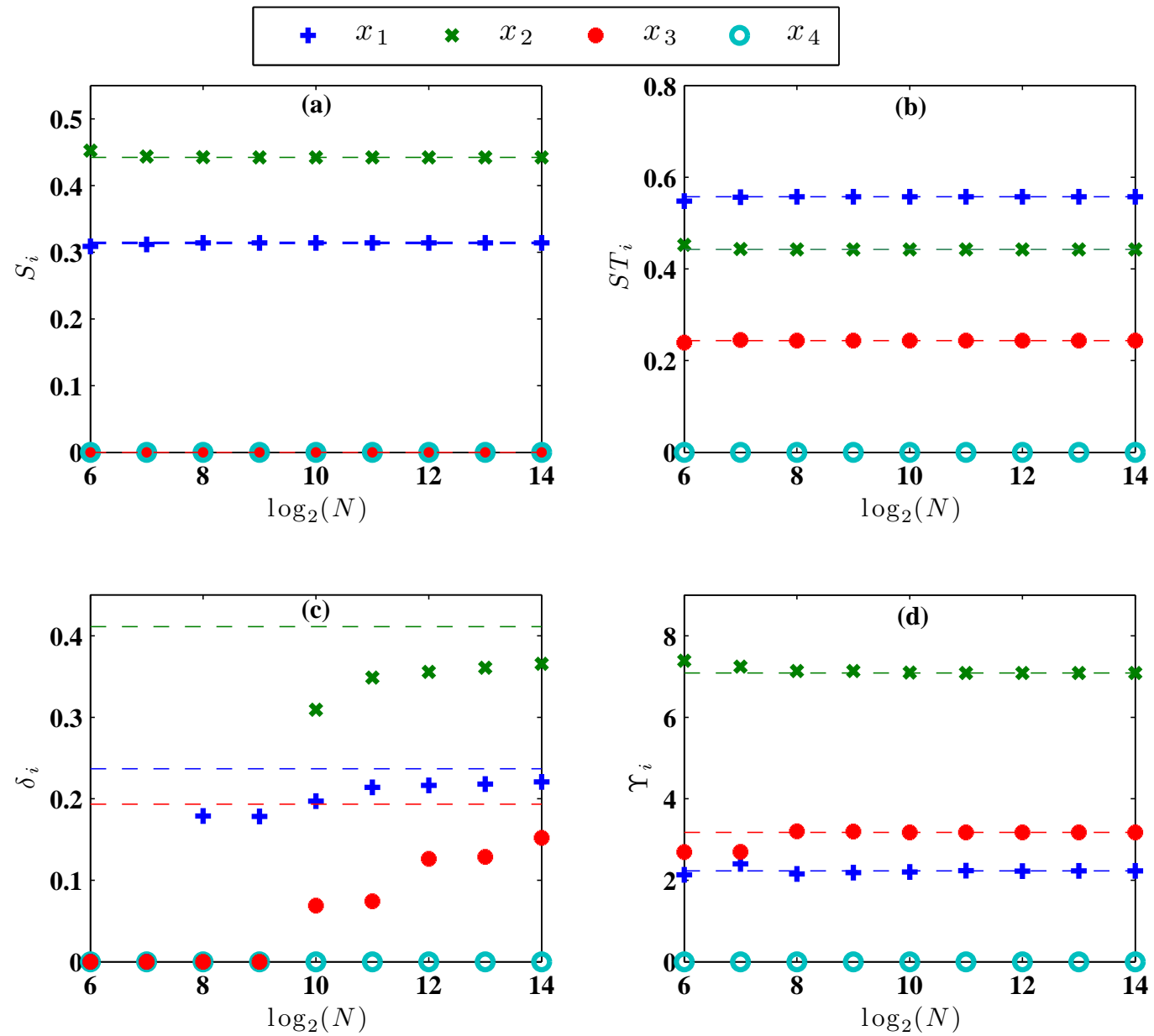

Figure 3: GSA of the Ishigami function - estimated sensitivity indices versus sample size.

The dashed lines are the reference values. 


\section{Application to an unsaturated flow model}

\subsection{Drainage experiment}

Modeling the flow in the unsaturated zone is crucial to predict groundwater resources evolution and forecast the migration of contaminants in aquifers. The hydraulic properties of soils are the key parameters controlling the flow through the unsaturated zone. They are generally estimated from laboratory drainage experiments. In such experiments, a flow cell filled with a saturated soil is drained by imposing a negative pressure head at the lower boundary of the cell. In the following, the global sensitivity methods are used to detect irrelevant hydraulic parameters of a synthetic laboratory drainage simulation.

To simulate the unsaturated flow, the Mualem-van Genuchten (MvG) retention curve is chosen to relate the hydraulic conductivity of the unsaturated soil to its water content (Mualem, 1976; van Genuchten, 1980). This retention curve is defined as follows:

$$
K=k_{s} \cdot S_{e}^{\lambda}\left(1-\left(1-S_{e}^{1 / m}\right)^{m}\right)^{2}
$$

where,

$$
S_{e}=\frac{\omega-\omega_{r}}{\omega_{s}-\omega_{r}}=\left\{\begin{array}{cc}
\frac{1}{\left(1+(\alpha|h|)^{n}\right)^{m}} & h<0 \\
1 & h \geq 0
\end{array}\right.
$$

with $m=1-1 / n$. The effective saturation $S_{e}$ links the pressure head $h[\mathrm{~cm}]$ to the water content $\omega\left[\mathrm{cm}^{3} \cdot \mathrm{cm}^{-3}\right]$ at any location in the soil.

The soil hydraulic parameters are: the saturated hydraulic conductivity $k_{s}\left[\mathrm{~m} \cdot \mathrm{s}^{-1}\right]$, the saturated water content $\omega_{s}\left[\mathrm{~cm}^{3} \cdot \mathrm{cm}^{-3}\right]$, the residual water content $\omega_{r}\left[\mathrm{~cm}^{3} \cdot \mathrm{cm}^{-3}\right]$ as well as the MvG parameters $\alpha\left[\mathrm{m}^{-1}\right], n[-]$ and $\lambda$ $[-]$. Hence, the random input vector is $\boldsymbol{x}=\left(k_{s}, \omega_{r}, \omega_{s}, \alpha, n, \lambda\right)$. 
The state variables (i.e. $h(t)$ and $\omega(t)$ ) are solution of the Richard's equation, that is,

$$
\frac{\partial \omega}{\partial t}=\frac{\partial}{\partial z}\left[K(h)\left(\frac{\partial h}{\partial z}-1\right)\right]
$$

where $t[\mathrm{~min}]$ is time and $z[\mathrm{~cm}]$ is the vertical coordinate (positive downward). This partial differential equation is solved with a standard Galerkin finite element method in conjunction with the Newton linearization method associated with the primary variable switching method (Diersch and Perrochet, 1999; Hayek et al., 2008).

For this numerical exercise we consider a cylindrical cell, $8.5 \mathrm{~cm}$ in diameter and $6 \mathrm{~cm}$ in height, filled with a soil. The parameters of the soil are assumed to be uniformly distributed over the ranges reported in Table 1. The soil, initially saturated with water, is drained by imposing a negative pressure head of $-700 \mathrm{~cm}$ in the bottom of the cell. The responses of interest are the pressure head $h$ at the center of the column, as well as the average soil water content $\omega_{\text {avg }}$ in the column after a simulation period of $250 \mathrm{~min}$. In addition to these responses, the numerical model provides the partial derivatives of these outputs with respect to the hydraulic parameters. Hence, there are four scalar responses computed by the numerical model. Preliminary studies showed that at least one thousand draws were necessary to obtain exploitable results with BIM. This finding is in agreement with the results of the Ishigami function (see the previous Section). Therefore, the computer model responses are evaluated for $N=1024$ different QMC draws.

\subsection{GSA of the pressure head}

First, let us analyse the results for the pressure head. The different sensitivity measure estimates are compiled in Table 1 . To assess the significance 
level of the sensitivity estimates, a dummy parameter has been included in the analysis. Its sensitivity indices were computed 1000 times, each time by assigning different random draws to the dummy parameter. The ranges of variation (minimal value-maximal value) of its estimated sensitivity indices are reported on the last column of Table 1 . We note that, all methods are in relative good agreement and indicate that $\left(k_{s}, \alpha, n\right)$ are the most important parameters.

A sparse PCE containing only 54 terms in an 11th-degree polynomial expansion has been built for the computation of the Sobol' indices. Comparison of first-order sensitivity indices with total sensitivity indices in Table 1 indicate that there are non-negligible interactions amongst the relevant parameters $\left(k_{s}, \alpha, n\right)$ (for these variables $\hat{S T_{i}^{P C E}}>\hat{S}_{i}^{P C E}$ ). This is one of the appealing features of variance-based sensitivity indices. Indeed, $\sum_{i=1}^{6} \hat{S}_{i}=0.86<1$ means that approximately $14 \%$ of the response variance is due to interactions amongst the input variables. According to the total sensitivity indices, the most important parameter is $k_{s}$, followed by $n$ and $\alpha$. The same ranking is observed when using first-order or total sensitivity indices. The contribution of the parameters $\omega_{r}, \omega_{s}$ and $\lambda$ to the total variance is insignificant (less than $2 \%)$.

The marginal effects of the hydraulic parameters onto the pressure head are depicted in Figure 4. They highlight the non-linear effects of $k_{s}$ and $n$ and the linear effect of $\alpha$. This figure also indicates that $k_{s}$ and $n$ have a monotonic decreasing effect onto the pressure head while $\alpha$ has an increasing effect. This means that increasing the value of $k_{s}$ alone (resp. $\alpha$ ) tends to decrease (resp. increase) the predicted value of the pressure head.

The density-based sensitivity measures are evaluated using a small number of partitions $(M=4)$, which provides subsamples of size $P=256$. The 
results in Table 1 show that the density-based sensitivity measures are able to detect the relevant inputs despite the small number of partitions. However, the ranking is different from that obtained with the Sobol' indices since the BIMs indicate that $\alpha$ is the most important parameter while $n$ is the least important one. It is worth noting that $\sum_{i=1}^{6} \hat{\delta}_{i}=0.7<1$, but in contrast with the first-order sensitivity indices, it does not mean that $30 \%$ of the output pdf is left unexplained by the BIMs. This is one drawback of Borgonovo's importance measure compared to the variance-based sensitivity measures, it does not provide any information about the model structure.

Both the indices $S T_{i}$ (Eq. (1b)) and $\delta_{i}$ (Eq. (8)) have the advantage of measuring the importance of the input variable regardless the nature of the model (linear or non-linear, with continuous values or not, etc.). However, with the variance-based measures, it is assumed that the response uncertainty is represented by its variance, while the density-based measure is momentindependent. Hence, the two sensitivity measures can provide different rankings regarding the order of importance of the input variables. In our opinion, $S T_{i}$ measures the importance of $x_{i}$ in the model structure while $\delta_{i}$ measures its importance for $p_{y}$. Regarding the objective of the study, that is, detecting the irrelevant inputs (FF setting), the two methods provide the same results: $\omega_{r}, \omega_{s}$ and $\lambda$ are not important parameters for predicting the pressure head.

The derivative-based global sensitivity indices are reported on the last row of Table 1. Obviously, these indices can be greater than one and in this case are not very informative. For instance, the DGSM of $\alpha\left(\hat{\Upsilon}_{\alpha}=0.36\right)$ should not be compared to that of $n\left(\hat{\Upsilon}_{n}=42.8\right)$ because DGSM is useful for screening the inputs and not for their ranking by order of importance. As expected, the DGSM is systematically greater than the respective total sensitivity index. This confirms that the DGSM is an upper bound for the 
total sensitivity index, that is, $S T_{i} \leq \Upsilon_{i}$. For the studied test case, it can be inferred that $\omega_{r}, \omega_{s}$ and $\lambda$ are irrelevant parameters for the predictive uncertainty of the pressure head.

\begin{tabular}{cccccccc}
\hline Parameter & $\begin{array}{c}k_{s} \\
{[\mathrm{~cm} / \mathrm{min}]}\end{array}$ & $\begin{array}{c}\omega_{r} \\
{\left[\mathrm{~cm}^{3} / \mathrm{cm}^{3}\right]}\end{array}$ & $\begin{array}{c}\omega_{s} \\
{\left[\mathrm{~cm}^{3} / \mathrm{cm}^{3}\right]}\end{array}$ & $\begin{array}{c}\alpha \\
{\left[\mathrm{cm}^{-1}\right]}\end{array}$ & $\begin{array}{c}n \\
{[-]}\end{array}$ & $\begin{array}{c}\lambda \\
{[-]}\end{array}$ & $\begin{array}{c}\text { dummy } \\
{[-]}\end{array}$ \\
\hline Range & {$[0.01,0.5]$} & {$[0.01,0.20]$} & {$[0.40,0.45]$} & {$[0.005,0.02]$} & {$[1.0,1.4]$} & {$[-0.5,1.0]$} & {$[-]$} \\
\hline$\hat{S}_{i}^{P C E}$ & 0.39 & 0.01 & 0.00 & 0.16 & 0.29 & 0.01 & {$[0.0,0.0]$} \\
\hline$\hat{S T}_{i}^{P C E}$ & 0.50 & 0.01 & 0.00 & 0.28 & 0.34 & 0.02 & {$[0.0,0.0]$} \\
\hline$\hat{\delta}_{i}$ & 0.23 & 0.06 & 0.00 & 0.26 & 0.10 & 0.05 & {$[0.0,0.08]$} \\
\hline$\hat{\Upsilon}_{i}$ & 6.94 & 0.02 & 0.00 & 0.36 & 42.8 & 0.02 & {$[0.0,0.0]$} \\
\hline
\end{tabular}

Table 1: GSA of the drainage model - estimated sensitivity indices for the pressure head. A dummy parameter is included to assess the significance of the estimated statistics (last column).

\subsection{GSA of the average water content}

The sensitivity indices for the average water content are gathered in Table 2. All sensitivity methods show that the parameter $n$ is by far the most important parameter.

The results of Table 2 indicate that the total sensitivity indices are close to the first-order indices $\left(\hat{S}_{i} \approx \hat{S T} T_{i}\right)$. This result means that the relationship between the model response and the parameters is virtually additive (i.e. with negligible interactions). The identified sparse PCE contained only 10 terms in a $2^{\text {nd }}$ degree polynomial expansion. The variance-based sensitivity indices point out the importance of $\left(\omega_{r}, \omega_{s}, \alpha, n\right)$ and the negligible influence of $k_{s}$ and $\lambda$ onto the average water content. The marginal effects plotted in Figure 5 highlight the linear effect of these parameters on this model response. 

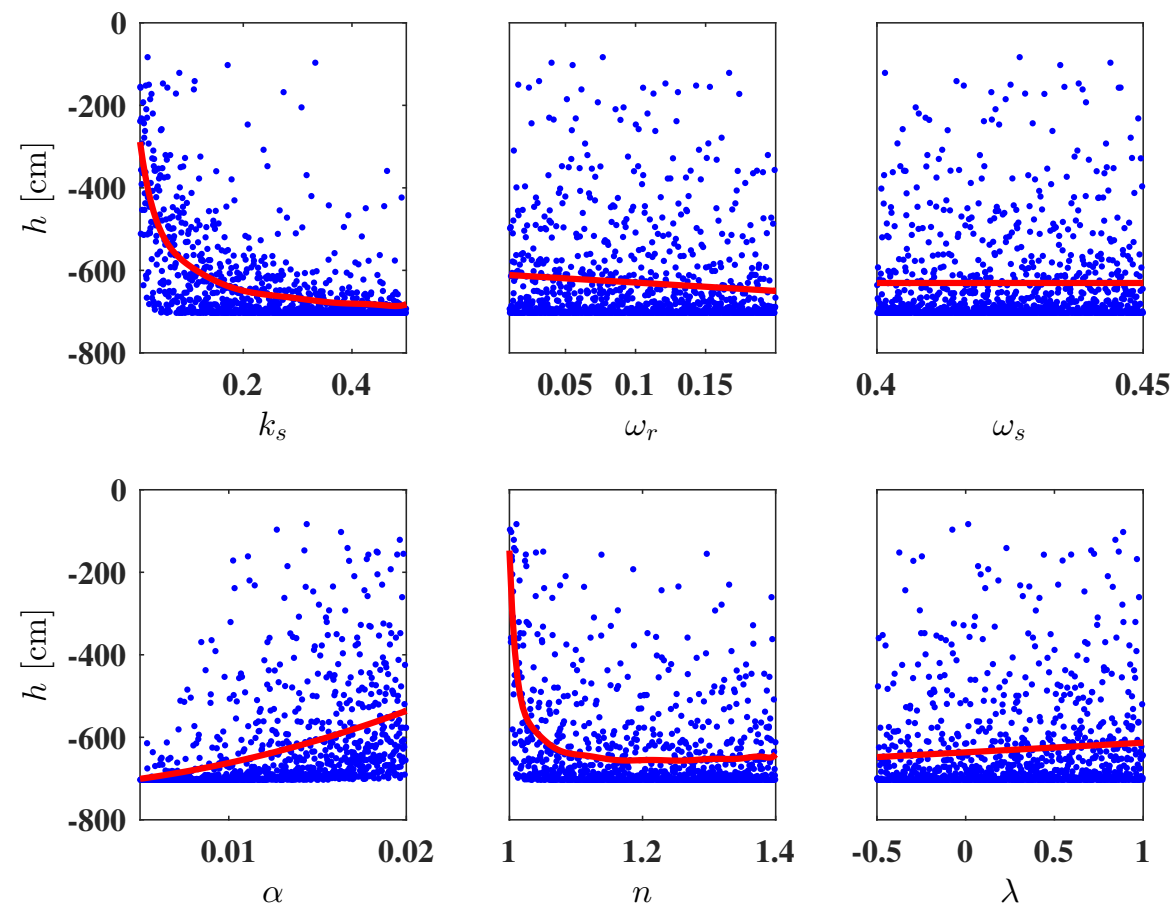

Figure 4: Marginal effects of the hydraulic parameters for the predicted pressure head. The red curve is the estimated conditional expectation (i.e. $\mathbb{E}\left[y \mid x_{i}\right]$ ) provided by the sparse PCE.

Contrary to the results for the pressure head, the variance and density based sensitivity rankings are in good agreement for the average water content. The ranking of importance is the same with the first-order sensitivity indices, the total sensitivity indices and the density-based sensitivity indices. The ranking of importance regarding the variance of the response indicates that the most important parameter is $n$, followed by $\omega_{r}$, then $\alpha$ and $\omega_{s}$.

Finally, the results of the DGSM must be interpreted with caution. As stated earlier, the DGSM cannot be used to rank the input variables by order of importance. The DGSM results in Table 2 show that the parameter $k_{s}$ is not considered to be irrelevant as found by all the other indicators. This 
result can be explained by analysing the scatterplot of the partial derivatives of the average water content with respect to $k_{s}$ in Figure 6 . This scatterplot depicts high local sensitivities in a very small region near the smallest value of $k_{s}$. However, this result does not mean that $k_{s}$ is globally a relevant parameter because the DGSM is an upper bound for the total sensitivity index which indicates that $k_{s}$ is irrelevant for the average water content (explaining only $1 \%$ of the variance). This result confirms that FF setting performed with the DGSM can be subject to Type I error.

\begin{tabular}{cccccccc}
\hline Parameter & $\begin{array}{c}k_{s} \\
{[\mathrm{~cm} / \mathrm{min}]}\end{array}$ & $\begin{array}{c}\omega_{r} \\
{\left[\mathrm{~cm}^{3} / \mathrm{cm}^{3}\right]}\end{array}$ & $\begin{array}{c}\omega_{s} \\
{\left[\mathrm{~cm}^{3} / \mathrm{cm}^{3}\right]}\end{array}$ & $\begin{array}{c}\alpha \\
{\left[\mathrm{cm}^{-1}\right]}\end{array}$ & $\begin{array}{c}n \\
{[-]}\end{array}$ & $\begin{array}{c}\lambda \\
{[-]}\end{array}$ & $\begin{array}{c}\text { dummy } \\
{[-]}\end{array}$ \\
\hline Range & {$[0.01,0.5]$} & {$[0.01,0.20]$} & {$[0.40,0.45]$} & {$[0.005,0.02]$} & {$[1.0,1.4]$} & {$[-0.5,1.0]$} & {$[-]$} \\
\hline$\hat{S}_{i}^{P C E}$ & 0.01 & 0.09 & 0.03 & 0.04 & 0.80 & 0.0 & {$[0.0,0.0]$} \\
\hline$\hat{S T}_{i}^{P C E}$ & 0.01 & 0.11 & 0.03 & 0.05 & 0.84 & 0.0 & {$[0.0,0.0]$} \\
\hline$\hat{\delta}_{i}$ & 0.0 & 0.09 & 0.04 & 0.06 & 0.44 & 0.0 & {$[0.0,0.04]$} \\
\hline$\hat{\Upsilon}_{i}$ & 0.32 & 0.14 & 0.04 & 0.09 & 1.10 & 0.0 & {$[0.0,0.0]$} \\
\hline
\end{tabular}

Table 2: GSA of the drainage model - estimated sensitivity indices for the average water content.

\section{Summary and conclusion}

In this paper, we discussed and tested three global sensitivity analysis methods that can be performed using only a single independent sample of modest size ( $\sim$ a thousand). The GSA methods allow to compute the following sensitivity indices: (i) the Sobol' indices, (ii) the Borgonovo measure of importance, and (iii) the derivative-based global sensitivity measure of Sobol' and Kucherenko. We tested the performances of the different methods for addressing factors fixing setting. This setting was applied to the Ishigami function and to a soil drainage model. 

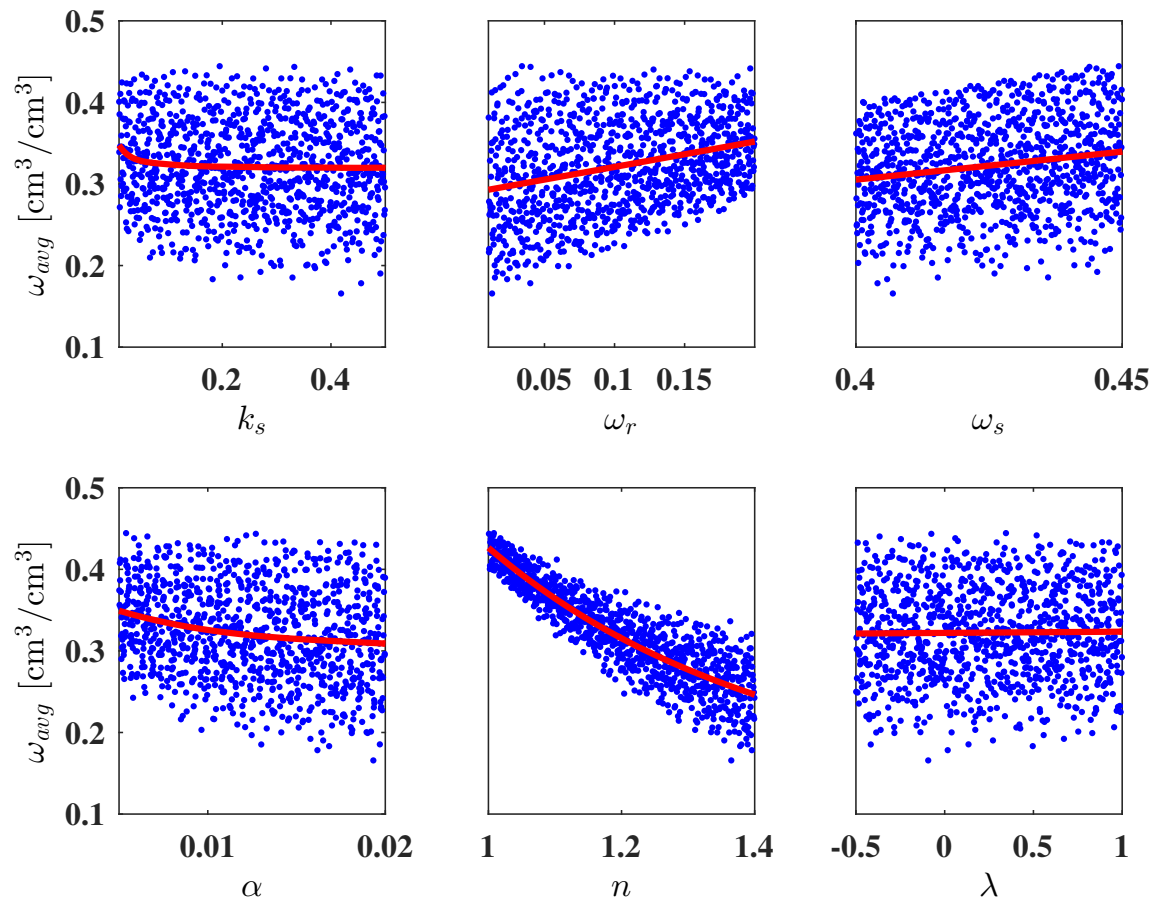

Figure 5: Marginal effects of the hydraulic parameters for the average water content. The red curve is the estimated conditional expectation (i.e. $\mathbb{E}\left[y \mid x_{i}\right]$ ) provided by the sparse PCE.

Variance-based sensitivity indices (also called Sobol' indices) are model free. They measure the amount of the model response variance due to each input variable alone and in interaction with the other variables. In the present work, they were computed by using the Bayesian sparse polynomial chaos expansion method developed by Shao et al. (2016). The results showed that this method provided reliable results at low computational cost. Moreover, PCE supplied quantitative insights into the input/output relationship.

Contrary to the Sobol' indices, the density-based sensitivity measure of Borgonovo is moment-independent. The BIMs were computed with the estimator proposed in Plischke et al. (2013). This estimator showed some prob- 

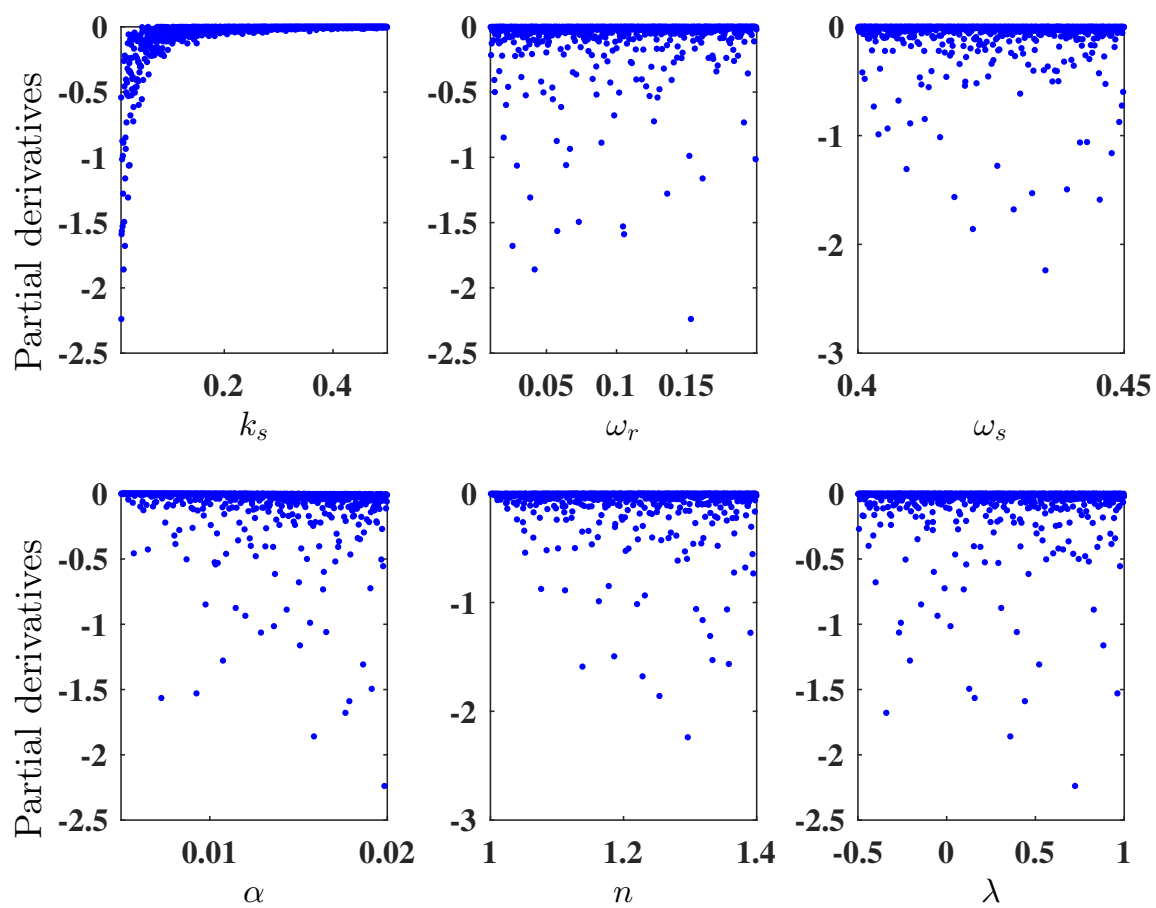

Figure 6: Partial derivatives of the average water content w.r.t. the hydraulic parameters.

lems of convergence of the estimated statistics at low sample sizes (see the Ishigami function). Despite of this problem, this method provided reliable results for FF setting if the sample size was sufficiently large ( $\sim$ a thousand).

The derivative-based global sensitivity measure (DGSM) is computed by evaluating the partial derivatives at several points in the input space. It requires that the computer model also provide the partial derivatives of the model response with respect to the input variables. The DGSM is an upper bound for the total sensitivity index. Thus, it cannot help in ranking the input variables by order of importance. However, the method is interesting for screening purposes that involve the investigation of unessential inputs (i.e. FF setting). However, our results proved that DGSM could be subject to Type I error, that is, identifying irrelevant inputs as possible relevant ones. 
In conclusion, GSA of computer models can be performed using different methods and free programs are often available for their computation when the inputs are independent. Several different global sensitivity measures can be computed from given data. In practice, the choice of the sensitivity measure to compute depends on the objective of the analysis. For the present work, it was found that sparse PCE was the most efficient method for addressing factors fixing setting.

Acknowledgment: This work has been funded by the French National Research Agency through the research project RESAIN (nANR-12-BS060010-02). The authors would like to thank the reviewers for their insightful comments that helped improving the manuscript. 


\section{References}

Anstett-Collin, F., Goffart, J., Mara, T., Denis-Vidal, L., 2015. Sensitivity analysis of complex models: Coping with dynamic and static inputs. Reliability Engineering and System Safety 134, 268-275.

Askey, R., Wilson, J., 1985. Some basic hypergeometric orthogonal polynomials that generalize Jacobi polynomials. Memoirs of the American Mathematical Society 54 (319), pp.55.

Blatman, G., Sudret, B., 2010. Efficient computation of global sensitivity indices using sparse polynomial chaos expansions. Reliability Engineering and System Safety 95 (11), 1216-1229.

Blatman, G., Sudret, B., 2011. Adaptive sparse polynomial chaos expansion based on least angle regression. Journal of Computational Physics 230 (6), $2345-2367$.

Borgonovo, E., 2006. Measuring uncertainty importance: Investigation and comparison of alternative approaches. Risk Analysis 26 (5), 1349-1361.

Borgonovo, E., 2007. A new uncertainty importance measure. Reliability Engineering and System Safety 92 (6), 771-784.

Borgonovo, E., Hazen, G. B., Plischke, E., 2016. A common rationale for global sensitivity measures and their estimation. Risk Analysis, 1-25.

Borgonovo, E., Plischke, E., 2015. Sensitivity analysis: A review of recent advances. European Journal of Operational Research, 1-19.

Cacuci, D., 1981. Sensitivity theory for nonlinear systems. I. nonlinear functional analysis approach. Journal of Mathematical Physics 82 (12), 27942802. 
Chun, M.-H., Han, S.-J., Tak, N.-I., 2000. An uncertainty importance measure using distance metric for the change in a cumulative distribution function. Reliability Engineering and System Safety 70, 313-321.

Cukier, R. I., Fortuin, C. M., Shuler, K. E., Petschek, A. G., Schaibly, J. H., 1973. Study of the sensitivity of coupled reaction systems to uncertainties in rate coefficients. I. theory. J. Chemical Physics 59, 3873-3878.

Diersch, H., Perrochet, P., 1999. On the primary variable switching technique for simulating unsaturated-saturated flows. Advances in Water Resources $23(3), 271-301$.

Fajraoui, N., Mara, T. A., Younes, A., Bouhlila, R., 2012. Reactive transport parameter estimation and global sensitivity analysis using sparse polynomial chaos expansion. Water, Air and Soil Pollution 223, 4183-4197.

Gamboa, F., Klein, T., Lagnoux, A., 2015. Sensitivity analysis based on cramer von mises distance. arXiv:1506.04133v1.

Gautschi, W., 1982. On generating orthogonal polynomials. SIAM Sci. Stat. Comput. 3 (3), 289-317.

Gautschi, W., 1993. Is the recurrence relation for orthogonal polynomials always stable? BIT 33, 277-284.

Griewank, W., 1989. On automatic differentiation. In: Mathematical programming: recent developments and applications. Kluwer Academic Publishers, 83-108.

Hayek, M., Lehmann, F., Ackerer, P., 2008. Adaptive multi-scale parameterization for one-dimensional flow in unsaturated porous media. Advances in Water Resources 31, 28-43. 
Homma, T., Saltelli, A., 1996. Importance measures in global sensitivity analysis of nonlinear models. Reliability Engineering and System Safety $52,1-17$.

Iooss, B., 2011. Revue sur l'analyse de sensibilité globale de modèles numériques. J. Société Française de Statistique 152 (1), 3-25.

Kucherenko, S., Tarantola, S., Annoni, P., 2012. Estimation of global sensitivity indices for models with dependent variables. Computer Physics Communications 183, 937-946.

Lamboni, M., Iooss, B., Popelin, A.-L., Gamboa, F., 2013. Derivative-based global sensitivity measures: general links with Sobol' indices and numerical tests. Mathematics and Computers in Simulation 87, 45-54.

Lewandowski, D., Cooke, R., Tebbens, R., 2007. Sample-based estimation of correlation ratio with polynomial approximation. ACM Transactions on Modeling and Computer Simulation 18 (1), 1-16.

Mara, T. A., Joseph, O. R., 2008. Comparison of some efficient methods to evaluate the main effect of computer model factors. Journal of Statistical Computation and Simulation 78, 167-178.

Mara, T. A., Tarantola, S., 2012. Variance-based sensitivity indices for models with dependent inputs. Reliability Engineering and System Safety 107, $115-121$.

Mara, T. A., Tarantola, S., Annoni, P., 2015. Non-parametric methods for global sensitivity analysis of model output with dependent inputs. Environmental Modelling and Software 72, 173-183. 
Marelli, S., Sudret, B., 2014. UQLab: A framework for uncertainty quantification in Matlab. In: Vulnerability, Uncertainty and Risk (2nd Int. Conf. on Vulnerability, Risk Analysis and Management). Liverpool, United Kingdom, pp. 2554-2563.

McKay, M. D., 1996. Variance-based methods for assessing uncertainty importance. Tech. rep., Technical Report NUREG-1150,UR-1996-2695, Los Alamos National Laboratory.

Mualem, Y., 1976. A new model for predicting the hydraulic conductivity of unsaturated porous media. Water Resources Research 12, 513-522.

Navon, I., 1997. Practical and theoretical aspect of adjoint parameter estimation and identifiability in meteorology and oceanography. Dynamics of Atmospheres and Oceans 27, 55-79.

Neumann, M. B., 2012. Comparison of sensitivity analysis methods for pollution degradation modelling: A case study from drinking water treatment. Science of Total Environment 433, 530-537.

Norton, J., 2015. An introduction to sensitivity assessment of simulation models. Environmental Modelling and Software 69, 166-174.

Oakley, J. E., O’Hagan, A., 2004. Probabilistic sensitivity analysis of complex models: a Bayesian approach. J. Royal Statist. Soc. B 66, 751-769.

Oladyshkin, S., Novak, W., 2012. Data-driven uncertainty quantification using the arbitrary polynomial chaos expansion. Reliability Engineering and System Safety 106, 179-190.

Owen, A. B., Dick, J., Chen, S., 2014. Higher order Sobol' indices. Information and Inference: A journal of IMA 3, 59-81. 
Parzen, E., 1962. On estimation of a probability density function and mode. Annals of Mathematical Statistics 33 (3), 1065-1076.

Pearson, K., 1905. On the general theory of skew correlation and non-linear regression. In: Mathematical contributions to the theory of evolution. Vol. XIV. Drapers's Company Research Memoirs.

Plischke, E., 2010. An effective algorithm for computing global sensitivity indices (EASI). Reliability Engineering and System Safety 95, 354-360.

Plischke, E., Borgonovo, E., Smith, C. L., 2013. Global sensitivity measures from given data. European Journal of Operational Research 226 (3), 536550 .

Press, W. H., Teukolsky, S. A., Vetterling, W. T., Flannery, B. P., 2007. Numerical Recipes: The Art of Scientific Computing. Cambridge University Press, N.Y. (third edition).

Rabitz, H., Alis, O., Shorter, J., Shim, K., 1999. Efficient input-output model representations. Computer Physics Communications 117, 11-20.

Ratto, M., Pagano, A., Young, P., 2007. State dependent parameter metamodelling and sensitivity analysis. Computer Physics Communications 117 (11), 863-876.

Saltelli, A., Ratto, M., Tarantola, S., Campolongo, F., 2006. Sensitivity analysis practices: Strategies for model-based inference. Reliability Engineering and System Safety 91, 1109-1125.

Saltelli, A., Ratto, M., Tarantola, S., Campolongo, F., 2012. Update 1 of: Sensitivity analysis for chemical models. Chemical Review 112 (5), PR1PR21. 
Saltelli, A., Tarantola, S., 2002. On the relative importance of input factors in mathematical models: Safety assessment for nuclear waste disposal. Journal of the American Statistical Association 97, 702-709.

Saltelli, A., Tarantola, S., Campolongo, F., Ratto, M., 2004. Sensitivity analysis in practice. Probability and Statistics. John Wiley and Sons, Chichester.

Saltelli, A., Tarantola, S., Chan, K., 1999. A quantitative model independent method for global sensitivity analysis of model output. Technometrics 41, $39-56$.

Schaibly, J., Shuler, K., 1973. Study of the sensitivity of coupled reaction systems to uncertainties in rate coefficients. II. Applications. J. Chemical Physics 59, 3879-3888.

Shao, Q., Younes, A., Fahs, M., Mara, T. A., 2016. Bayesian sparse polynomial chaos expansion for global sensitivity analysis. submitted to Computational Methods in Applied Mechanical Engineering, under review.

Sobol', I. M., 1993. Sensitivity estimates for nonlinear mathematical models. Math. Mod. and Comput. Exp. 1, 407-414.

Sobol', I. M., Kucherenko, S., 2009. Derivative based global sensitivity measures and their link with global sensitivity indices. Mathematics and Computers in Simulation 79, 3009-3017.

Sobol', I. M., Kucherenko, S., 2010. A new derivative based importance criterion for groups of variables and its link with the global sensitivity indices. Computer Physics Communications 181, 1212-1217. 
Sobol', I. M., Turchaninov, V. I., Levitan, Y. L., Shukman, B. V., 1992. Quasi-random sequence generator (routine LPTAU51). Keldysh Institute of Applied Mathematics, Russian Academy of Sciences.

Strong, M., Oakley, J. E., 2013. An efficient method for computing partial expected value of perfect information. Medical Decision-Making 33, 755766.

Sudret, B., 2008. Global sensitivity analysis using polynomial chaos expansions. Reliability Engineering and System Safety 93, 964-979.

Tarantola, S., Gatelli, D., Mara, T. A., 2006. Random balance designs for the estimation of first-order global sensitivity indices. Reliability Engineering and System Safety 91, 717-727.

van Genuchten, M. T., 1980. A closed form equation for predicting the hydraulic properties of unsaturated soils. Soil Science Society of America Journal 44 (5), 892-898.

Xiu, D., Karniadakis, G. E., 2002. The Wiener-Askey polynomial chaos for stochastic differential equations. SIAM, Journal of Scientific Computing 24, 619-644. 